\title{
RELIABILITY FOR SOME BIVARIATE GAMMA DISTRIBUTIONS
}

\author{
SARALEES NADARAJAH
}

Received 23 July 2004 and in revised form 23 November 2004

In the area of stress-strength models, there has been a large amount of work as regards estimation of the reliability $R=\operatorname{Pr}(X<Y)$. The algebraic form for $R=\operatorname{Pr}(X<Y)$ has been worked out for the vast majority of the well-known distributions when $X$ and $Y$ are independent random variables belonging to the same univariate family. In this paper, we consider forms of $R$ when $(X, Y)$ follows a bivariate distribution with dependence between $X$ and $Y$. In particular, we derive explicit expressions for $R$ when the joint distribution is bivariate gamma. The calculations involve the use of special functions.

\section{Introduction}

Bivariate gamma distributions arise as tractable "lifetime" models in many areas, including life testing and telecommunications. In the context of reliability, the stress-strength model describes the life of a component which has a random strength $Y$ and is subjected to a random stress $X$. The component fails at the instant that the stress applied to it exceeds the strength and the component will function satisfactorily whenever $Y>X$. Thus, $R=\operatorname{Pr}(X<Y)$ is a measure of the component reliability. It has many applications especially in engineering concepts such as structures, deterioration of rocket motors, static fatigue of ceramic components, fatigue failure of aircraft structures, and the aging of concrete pressure vessels. Some examples are as follows.

(i) If $X$ represents the maximum chamber pressure generated by ignition of a solid propellant and $Y$ represents the strength of the rocket chamber, then $R$ is the probability of successful firing of the engine.

(ii) If $X$ represents the diameter of a shaft and $Y$ represents the diameter of a bearing that is to be mounted on the shaft, then $R$ is the probability that the bearing fits without interference.

(iii) Let $Y$ and $X$ be the remission times of two chemicals when they are administered to two kinds of mechanical systems. Inferences about $R$ present a comparison of the effectiveness of the two chemicals.

(iv) If $X$ and $Y$ are future observations on the stability of an engineering design, then $R$ would be the predictive probability that $X$ is less than $Y$. Similarly, if $X$ and 
152 Reliability for some bivariate gamma distributions

$Y$ represent lifetimes of two electronic devices, then $R$ is the probability that one fails before the other.

(v) If $Y$ represents the distance of a pyrotechnic igniter from its adjacent pellet and $X$ represents its ignition distance, then $R$ is the probability that the igniter succeeds to bridge the gap in the pyrotechnic chain.

(vi) A certain unit-be it a receptor in a human eye or ear or any other organ (including sexual) - operates only if it is stimulated by the source of random magnitude $Y$ and the stimulus exceeds a lower threshold $X$ specific for that unit. In this case, $R$ is the probability that the unit functions.

(vii) In military warfare, $R$ could be interpreted as the probability that a given round of ammunition will penetrate its target.

Because of these applications, the calculation and the estimation of $R=\operatorname{Pr}(X<Y)$ are important for the class of bivariate gamma distributions. The calculation of $R$ has been extensively investigated in the literature when $X$ and $Y$ are independent random variables belonging to the same univariate family of distributions. The algebraic form for $R$ has been worked out for the majority of the well-known distributions, including normal, uniform, exponential, gamma, beta, extreme value, Weibull, Laplace, logistic, and the Pareto distributions (Nadarajah [21, 22, 23, 24, 25]; Nadarajah and Kotz [27]). However, there is relative little work when $X$ and $Y$ are dependent random variables. A complete literature search shows that the cases of bivariate beta (Nadarajah [26]), bivariate exponential (Awad et al. [2], Klein and Basu [18], Jana [13], Jana and Roy [15], Jana [14], Jeevanand [17], Hanagal [10, 12]), bivariate normal (Mukherjee and Saran [20], Nandi and Aich [28, 29], Gupta and Subramanian [8]), and bivariate Pareto (Hanagal [11], Jeevanand [16]) have been considered. The cases of multivariate exponential (Cramer and Kamps [5]) and multivariate normal (Singh [33], R. D. Gupta and R. C. Gupta [9]) have also been considered. We have been able to trace no other work for dependent variables.

The aim of this paper is to calculate $R$ when $X$ and $Y$ are dependent random variables from six flexible families of bivariate gamma distributions. We will assume throughout this paper that $(X, Y)$ has a bivariate gamma distribution with joint probability density function (pdf) $f$ and joint survivor function $\bar{F}$. One can write

$$
R=\int_{0}^{\infty} \int_{x}^{\infty} f(x, y) d y d x
$$

Our calculations of $R$ make use of a number of special functions. They are the complementary incomplete gamma function defined by

$$
\Gamma(a, x)=\int_{x}^{\infty} t^{a-1} \exp (-t) d t
$$

the confluent hypergeometric function $\left({ }_{1} F_{1}\right)$ defined by

$$
{ }_{1} F_{1}(a ; b ; x)=\sum_{k=0}^{\infty} \frac{(a)_{k}}{(b)_{k}} \frac{x^{k}}{k !},
$$


the Gauss hypergeometric function $\left({ }_{2} F_{1}\right)$ defined by

$$
{ }_{2} F_{1}(a, b ; c ; x)=\sum_{k=0}^{\infty} \frac{(a)_{k}(b)_{k}}{(c)_{k}} \frac{x^{k}}{k !},
$$

the generalized hypergeometric function $\left({ }_{2} F_{2}\right)$ defined by

$$
{ }_{2} F_{2}(a, b ; c, d ; x)=\sum_{k=0}^{\infty} \frac{(a)_{k}(b)_{k}}{(c)_{k}(d)_{k}} \frac{x^{k}}{k !},
$$

the Kummer function defined by

$$
\Psi(a, b, x)=\frac{1}{\Gamma(a)} \int_{0}^{\infty} t^{a-1}(1+t)^{b-a-1} \exp (-z t) d t
$$

and the modified Bessel function of the first kind defined by

$$
I_{m}(z)=\sum_{k=0}^{\infty} \frac{z^{2 k+m}}{2^{2 k+m} k ! \Gamma(k+m+1)},
$$

where $(e)_{k}=e(e+1) \cdots(e+k-1)$ denotes the ascending factorial. The properties of these special functions being used can be found in Prudnikov et al. [30, 31, 32] and Gradshteyn and Ryzhik [7].

The paper is organized as follows. Sections 2 to 7 calculate expressions for $R$ for the six distributions (Section 2 for McKay's bivariate gamma distribution, Section 3 for Dussauchoy and Berland's bivariate gamma distribution, Section 4 for Cherian's bivariate gamma distribution, Section 5 for Arnold and Strauss' bivariate gamma distribution, Section 6 for Becker and Roux's bivariate gamma distribution, and Section 7 for Smith and Adelfang's bivariate gamma distribution.) Conclusions and suggestions for future work are described in Section 8.

\section{McKay's bivariate gamma distribution}

McKay's [19] bivariate gamma distribution has the joint pdf given by

$$
f(x, y)=\frac{a^{p+q}}{\Gamma(p) \Gamma(q)} x^{p-1}(y-x)^{q-1} \exp (-a y)
$$

for $y>x>0, a>0, p>0$, and $q>0$. For this distribution, it is easily seen that $R$ is given by

$$
\begin{aligned}
R & =\frac{a^{p+q}}{\Gamma(p) \Gamma(q)} \int_{0}^{\infty} x^{p-1} \int_{x}^{\infty}(y-x)^{q-1} \exp (-a y) d y d x \\
& =\frac{a^{p+q}}{\Gamma(p) \Gamma(q)} \int_{0}^{\infty} x^{p-1} \Gamma(q) d x \\
& =a^{p+q} .
\end{aligned}
$$


154 Reliability for some bivariate gamma distributions

\section{Dussauchoy and Berland's bivariate gamma distribution}

Dussauchoy and Berland's [6] bivariate gamma distribution has the joint pdf specified by

$$
\begin{aligned}
f(x, y)= & \frac{\beta a_{2}^{q}}{\Gamma(p) \Gamma(q-p)}(\beta x)^{p-1}(y-\beta x)^{q-p-1} \exp \left(-a_{2} x\right) \exp \left\{-\frac{a_{2}}{\beta}(y-\beta x)\right\} \\
& \times{ }_{1} F_{1}\left(p, q-p ;\left(\frac{a_{1}}{\beta}-a_{2}\right)(y-\beta x)\right)
\end{aligned}
$$

for $x>0, y>0,0 \leq \beta \leq 1,0<a_{2} \leq a_{1} / \beta$, and $0<p<q$. Using the definition of the confluent hypergeometric function given in Section 1, one can express the corresponding form of $R$ as

$$
\begin{aligned}
& R=\frac{\beta a_{2}^{q}}{\Gamma(p) \Gamma(q-p)} \int_{0}^{\infty}(\beta x)^{p-1} \exp \left(-a_{2} x\right) \\
& \times \int_{x}^{\infty}(y-\beta x)^{q-p-1} \exp \left\{-\frac{a_{2}}{\beta}(y-\beta x)\right\} \\
& \times \sum_{k=0}^{\infty} \frac{(p)_{k}}{k !(q-p)_{k}}\left(\frac{a_{1}}{\beta}-a_{2}\right)^{k}(y-\beta x)^{k} d y d x \\
& =\frac{\beta a_{2}^{q}}{\Gamma(p) \Gamma(q-p)} \int_{0}^{\infty}(\beta x)^{p-1} \exp \left(-a_{2} x\right) \sum_{k=0}^{\infty} \frac{(p)_{k}}{k !(q-p)_{k}}\left(\frac{a_{1}}{\beta}-a_{2}\right)^{k} \\
& \times \int_{x}^{\infty}(y-\beta x)^{k+q-p-1} \exp \left\{-\frac{a_{2}}{\beta}(y-\beta x)\right\} d y d x \\
& =\frac{\beta^{q} a_{2}^{p}}{\Gamma(p) \Gamma(q-p)} \int_{0}^{\infty} x^{p-1} \exp \left(-a_{2} x\right) \sum_{k=0}^{\infty} \frac{(p)_{k}}{k !(q-p)_{k}}\left(\frac{a_{1}}{a_{2}}-\beta\right)^{k} \\
& \times \int_{a_{2}(1-\beta) x / \beta}^{\infty} w^{k+q-p-1} \exp (-w) d w d x \\
& =\frac{\beta^{q} a_{2}^{p}}{\Gamma(p) \Gamma(q-p)} \int_{0}^{\infty} x^{p-1} \exp \left(-a_{2} x\right) \sum_{k=0}^{\infty} \frac{(p)_{k}}{k !(q-p)_{k}}\left(\frac{a_{1}}{a_{2}}-\beta\right)^{k} \\
& \times \Gamma\left(k+q-p, \frac{a_{2}(1-\beta) x}{\beta}\right) d x \\
& =\frac{\beta^{q} a_{2}^{p}}{\Gamma(p) \Gamma(q-p)} \sum_{k=0}^{\infty} \frac{(p)_{k}}{k !(q-p)_{k}}\left(\frac{a_{1}}{a_{2}}-\beta\right)^{k} I(k),
\end{aligned}
$$

where the transformation $w=a_{2}(y-\beta x) / \beta$ has been applied and $I(k)$ denotes the integral

$$
I(k)=\int_{0}^{\infty} x^{p-1} \exp \left(-a_{2} x\right) \Gamma\left(k+q-p, \frac{a_{2}(1-\beta) x}{\beta}\right) d x
$$


Application of Lemma A.1 in the appendix shows that $I(k)$ can be expressed in terms of the Gauss hypergeometric function as

$$
I(k)=\frac{\beta^{p}(1-\beta)^{k+q}}{p a_{2}^{p}} \Gamma(k+q){ }_{2} F_{1}(1, k+q ; p+1 ; \beta) .
$$

If $q-p$ is an integer, then by using the property

$$
\Gamma(n, z)=(n-1) ! \exp (-z) \sum_{m=0}^{n-1} \frac{z^{k}}{k !}
$$

one can obtain the elementary expression

$$
\begin{aligned}
I(k) & =\int_{0}^{\infty} x^{p-1} \exp \left(-a_{2} x\right)(k+q-p-1) ! \sum_{m=0}^{k+q-p-1} \frac{1}{m !}\left\{\frac{a_{2}(1-\beta) x}{\beta}\right\}^{m} d x \\
& =(k+q-p-1) ! \sum_{m=0}^{k+q-p-1} \frac{1}{m !}\left\{\frac{a_{2}(1-\beta)}{\beta}\right\}^{m} \int_{0}^{\infty} x^{m+p-1} \exp \left(-a_{2} x\right) d x \\
& =(k+q-p-1) !\left(\frac{a_{2}}{\beta}\right)^{p} \sum_{m=0}^{k+q-p-1} \frac{\Gamma(p+m)(1-\beta)^{m}}{m !} .
\end{aligned}
$$

Expressions for $R$ can be obtained by substituting (3.4) and (3.6) into (3.2).

\section{Cherian's bivariate gamma distribution}

Cherian's [4] bivariate gamma distribution has the joint pdf specified by

$$
f(x, y)=K \exp (-x) \exp (-y) \int_{0}^{\min (x, y)}(x-z)^{\theta_{1}-1}(y-z)^{\theta_{2}-1} z^{\theta_{3}-1} \exp (z) d z
$$

for $x>0, y>0, \theta_{1}>0, \theta_{2}>0$, and $\theta_{3}>0$, where $K$ denotes the normalizing constant given by

$$
\frac{1}{K}=\Gamma\left(\theta_{1}\right) \Gamma\left(\theta_{2}\right) \Gamma\left(\theta_{3}\right)
$$

For this distribution, the form of $R$ can be expressed as

$$
\begin{aligned}
R & =K \int_{0}^{\infty} \int_{x}^{\infty} \int_{0}^{\min (x, y)}(x-z)^{\theta_{1}-1}(y-z)^{\theta_{2}-1} z^{\theta_{3}-1} \exp (z-x-y) d z d y d x \\
& =K \int_{0}^{\infty} \int_{0}^{x}(x-z)^{\theta_{1}-1} \exp (-x) z^{\theta_{3}-1} \exp (z)\left\{\int_{x}^{\infty}(y-z)^{\theta_{2}-1} \exp (-y) d y\right\} d z d x \\
& =K \int_{0}^{\infty} \int_{0}^{x}(x-z)^{\theta_{1}-1} \exp (-x) z^{\theta_{3}-1} \exp (z)\left\{\int_{x-z}^{\infty} w^{\theta_{2}-1} \exp (-w) d w\right\} d z d x
\end{aligned}
$$


156 Reliability for some bivariate gamma distributions

$$
\begin{aligned}
& =K \int_{0}^{\infty} \int_{0}^{x}(x-z)^{\theta_{1}-1} \exp (-x) z^{\theta_{3}-1} \Gamma\left(\theta_{2}, x-z\right) d z d x \\
& =K \int_{0}^{\infty} \int_{0}^{x}(x-z)^{\theta_{1}-1} \exp (-x) z^{\theta_{3}-1} \Gamma\left(\theta_{2}, x-z\right) d z d x \\
& =K \int_{0}^{\infty} x^{\theta_{1}+\theta_{3}-1} \exp (-x) \int_{0}^{1} t^{\theta_{1}-1}(1-t)^{\theta_{3}-1} \Gamma\left(\theta_{2}, x t\right) d t d x,
\end{aligned}
$$

where the transformations $w=y-z$ and $t=(x-z) / x$ have been applied. Application of Lemma A.2 shows that the inner integral in (4.3) can be calculated as

$$
\begin{aligned}
& \int_{0}^{1} t^{\theta_{1}-1}(1-t)^{\theta_{3}-1} \Gamma\left(\theta_{2}, x t\right) d t \\
& \quad=\Gamma\left(\theta_{2}\right) B\left(\theta_{1}, \theta_{3}\right)+\theta_{2}^{-1} B\left(\theta_{3}, \theta_{1}+\theta_{2}\right) x^{\theta_{2}}{ }_{2} F_{2}\left(\theta_{2}, \theta_{1}+\theta_{2} ; \theta_{2}+1 ; \theta_{1}+\theta_{2}+\theta_{3} ;-x\right),
\end{aligned}
$$

and thus $R$ can be rewritten as

$$
R=\frac{1}{\Gamma\left(\theta_{1}\right) \Gamma\left(\theta_{3}\right)}+\theta_{2}^{-1} B\left(\theta_{3}, \theta_{1}+\theta_{2}\right) I
$$

where $I$ denotes the integral

$$
I=\int_{0}^{\infty} x^{\theta_{1}+\theta_{2}+\theta_{3}-1} \exp (-x)_{2} F_{2}\left(\theta_{2}, \theta_{1}+\theta_{2} ; \theta_{2}+1 ; \theta_{1}+\theta_{2}+\theta_{3} ;-x\right) d x
$$

This integral can be calculated by an application of Lemma A.3 to yield

$$
I=\Gamma\left(\theta_{1}+\theta_{2}+\theta_{3}\right)_{2} F_{1}\left(\theta_{2}, \theta_{1}+\theta_{2} ; \theta_{2}+1 ;-1\right),
$$

and hence it follows from (4.5) that the form of $R$ for Cherian's bivariate gamma distribution is given by

$$
R=1+\theta_{2}^{-1} \Gamma\left(\theta_{1}+\theta_{2}\right) \Gamma\left(\theta_{3}\right){ }_{2} F_{1}\left(\theta_{2}, \theta_{1}+\theta_{2} ; \theta_{2}+1 ;-1\right) .
$$

\section{Arnold and Strauss' bivariate gamma distribution}

Arnold and Strauss's [1] bivariate gamma distribution has the joint pdf specified by

$$
f(x, y)=K x^{\alpha-1} y^{\beta-1} \exp \{-(a x+b y+c x y)\}
$$

for $x>0, y>0, \alpha>0, \beta>0, a>0, b>0$, and $c>0$, where $K=K(a, b, c, \alpha, \beta)$ denotes the normalizing constant given by

$$
\frac{1}{K}=b^{\alpha-\beta} c^{-\alpha} \Gamma(\alpha) \Gamma(\beta) \Psi\left(\alpha, \alpha-\beta+1, \frac{a b}{c}\right) .
$$


For this distribution, the form of $R$ can be derived as follows:

$$
\begin{aligned}
R & =K \int_{0}^{\infty} x^{\alpha-1} \exp (-a x) \int_{x}^{\infty} y^{\beta-1} \exp \{-(b+c x) y\} d y d x \\
& =K \int_{0}^{\infty} \frac{x^{\alpha-1} \exp (-a x)}{(b+c x)^{\beta}} \int_{(b+c x) x}^{\infty} z^{\beta-1} \exp (-z) d z d x \\
& =K \int_{0}^{\infty} \frac{x^{\alpha-1} \exp (-a x) \Gamma(\beta,(b+c x) x)}{(b+c x)^{\beta}} d x
\end{aligned}
$$

where the transformation $z=(b+c x) y$ has been applied. The integral in (5.3) cannot be simplified further into its general form. However, if $\beta$ is an integer, then using (3.5) one can rewrite (5.3) as

$$
\begin{aligned}
R & =K(\beta-1) ! \int_{0}^{\infty} \frac{x^{\alpha-1} \exp (-a x)}{(b+c x)^{\beta}} \exp \{-(b+c x) x\} \sum_{l=0}^{\beta-1} \frac{(b+c x)^{l} x^{l}}{l !} d x \\
& =K(\beta-1) ! \sum_{l=0}^{\beta-1} \frac{I(l)}{l !}
\end{aligned}
$$

where $I(l)$ denotes the integral

$$
I(l)=\int_{0}^{\infty}(b+c x)^{l-\beta} x^{l+\alpha-1} \exp \left\{-\left(a x+b x+c x^{2}\right)\right\} d x
$$

Again, this integral cannot be simplified further unless $\alpha$ is also an integer. If this is assumed then-by transforming $y=b+c x$-one can express

$$
\begin{aligned}
I(l)= & c^{-(l+\alpha)} \exp \left\{\frac{(a+b)^{2}}{4 c}\right\} \int_{b}^{\infty} y^{l-\beta}(y-b)^{l+\alpha-1} \exp \left\{-\frac{1}{c}\left(y+\frac{a-b}{2}\right)^{2}\right\} d y \\
= & c^{-(l+\alpha)} \exp \left\{\frac{(a+b)^{2}}{4 c}\right\} \int_{b}^{\infty} y^{l-\beta}\left[\sum_{m=0}^{l+\alpha-1}\left(\begin{array}{c}
l+\alpha-1 \\
m
\end{array}\right)(-b)^{l+\alpha-1-m} y^{m}\right] \\
& \times \exp \left\{-\frac{1}{c}\left(y+\frac{a-b}{2}\right)^{2}\right\} d y \\
= & c^{-(l+\alpha)} \exp \left\{\frac{(a+b)^{2}}{4 c}\right\} \sum_{m=0}^{l+\alpha-1}\left(\begin{array}{c}
l+\alpha-1 \\
m
\end{array}\right)(-b)^{l+\alpha-1-m} J(m, l),
\end{aligned}
$$

where

$$
J(m, l)=\int_{b}^{\infty} y^{m-\beta+l} \exp \left\{-\frac{1}{c}\left(y+\frac{a-b}{2}\right)^{2}\right\} d y .
$$


158 Reliability for some bivariate gamma distributions

The integral $J(m, l)$ can be easily calculated. Three cases need to be considered.

(1) If $m=\beta-l$, then

$$
J(m, l)=\sqrt{\pi c}\left\{1-\Phi\left(\frac{a+b}{\sqrt{2 c}}\right)\right\}
$$

where $\Phi(\cdot)$ denotes the cdf of the standard normal distribution.

(2) If $m>\beta-l$, then-by transforming $z=\{y+(a-b) / 2\}^{2}$-one can write

$$
\begin{aligned}
J(m, l) & =\frac{1}{2} \int_{(a+b)^{2} / 4}^{\infty} \frac{1}{\sqrt{z}}\left(\sqrt{z}+\frac{b-a}{2}\right)^{m-\beta+l} \exp \left(-\frac{z}{c}\right) d z \\
& =\frac{1}{2} \int_{(a+b)^{2} / 4}^{\infty} \frac{1}{\sqrt{z}}\left\{\sum_{n=0}^{m-\beta+l}\left(\begin{array}{c}
m-\beta+l \\
n
\end{array}\right)\left(\frac{b-a}{2}\right)^{m-\beta+l-n} z^{n / 2}\right\} \exp \left(-\frac{z}{c}\right) d z \\
& =\frac{1}{2} \sum_{n=0}^{m-\beta+l}\left(\begin{array}{c}
m-\beta+l \\
n
\end{array}\right)\left(\frac{b-a}{2}\right)^{m-\beta+l-n} \int_{(a+b)^{2} / 4}^{\infty} z^{(n-1) / 2} \exp \left(-\frac{z}{c}\right) d z \\
& =\frac{1}{2} \sum_{n=0}^{m-\beta+l}\left(\begin{array}{c}
m-\beta+l \\
n
\end{array}\right)\left(\frac{b-a}{2}\right)^{m-\beta+l-n} c^{(n+1) / 2} \Gamma\left(\frac{n+1}{2}, \frac{(a+b)^{2}}{4 c}\right),
\end{aligned}
$$

a finite sum of incomplete gamma functions.

(3) If $m<\beta-l$, then the same transformation as above yields

$$
\begin{aligned}
J(m, l) & =\frac{1}{2} \int_{(a+b)^{2} / 4}^{\infty} z^{(m-\beta+l-1) / 2}\left(1+\frac{b-a}{2 \sqrt{z}}\right)^{m-\beta+l} \exp \left(-\frac{z}{c}\right) d z \\
& =\frac{1}{2} \int_{(a+b)^{2} / 4}^{\infty} z^{(m-\beta+l-1) / 2}\left\{\sum_{n=0}^{\infty}\left(\begin{array}{c}
m-\beta+l \\
n
\end{array}\right)\left(\frac{b-a}{2 \sqrt{z}}\right)^{n}\right\} \exp \left(-\frac{z}{c}\right) d z \\
& =\frac{1}{2} \sum_{n=0}^{\infty}\left(\begin{array}{c}
m-\beta+l \\
n
\end{array}\right)\left(\frac{b-a}{2}\right)^{n} \int_{(a+b)^{2} / 4}^{\infty} z^{(m-\beta+l-n-1) / 2} \exp \left(-\frac{z}{c}\right) d z \\
& =\frac{1}{2} \sum_{n=0}^{\infty}\left(\begin{array}{c}
m-\beta+l \\
n
\end{array}\right)\left(\frac{b-a}{2}\right)^{n} c^{(m-\beta+l-n+1) / 2} \times \Gamma\left(\frac{m-\beta+l-n+1}{2}, \frac{(a+b)^{2}}{4 c}\right),
\end{aligned}
$$

an infinite sum of incomplete gamma functions.

Combining (5.4)-(5.10), the reliability $R$ can be expressed as an infinite sum of incomplete gamma functions for integer values of $\alpha$ and $\beta$ and general $a, b$, and $c$. However, if $a=b$, then one can express $R$ as a finite sum of incomplete gamma functions. This follows because if $a=b$, then $J(m, l)$ reduces to

$$
\begin{aligned}
J(m, l) & =2^{-1} c^{1+(m-\beta) / 2} \int_{a^{2} / c}^{\infty} z^{(m-\beta) / 2} \exp (-z) d z \\
& =2^{-1} c^{1+(m-\beta) / 2} \Gamma\left(1+\frac{m-\beta}{2}, \frac{a^{2}}{c}\right)
\end{aligned}
$$


(after substituting $z=y^{2} / c$ ), and combining (5.11) with (5.4)-(5.6), one obtains

$$
R=\frac{K(\beta-1) ! \exp \left(a^{2} / c\right)}{2(-a)^{1-\alpha} c^{\alpha+\beta / 2-1}} \sum_{l=0}^{\beta-1} \sum_{m=0}^{l+\alpha-1} \frac{(-a)^{l-m}}{l ! c^{l-m / 2}}\left(\begin{array}{c}
l+\alpha-1 \\
m
\end{array}\right) \Gamma\left(1+\frac{m-\beta}{2}, \frac{a^{2}}{c}\right),
$$

a finite sum of incomplete gamma functions. If also $\alpha=\beta$, then it is easy to see that $R=1 / 2$.

\section{Becker and Roux's bivariate gamma distribution}

Becker and Roux's [3] bivariate gamma distribution has the joint pdf given by

$$
\begin{aligned}
& f(x, y) \\
& \quad= \begin{cases}\frac{\lambda_{2}}{\Gamma(h) \Gamma(l) \alpha_{1}^{h} \alpha_{2}^{l}} x^{h-1}\left\{\lambda_{2}(y-x)+x\right\}^{l-1} \exp \left\{-\left(\frac{1}{\alpha_{1}}+\frac{1-\lambda_{2}}{\alpha_{2}}\right) x-\frac{\lambda_{2}}{\alpha_{2}} y\right\} & \text { if } x<y, \\
\frac{\lambda_{1}}{\Gamma(h) \Gamma(l) \alpha_{1}^{h} \alpha_{2}^{l}} y^{l-1}\left\{\lambda_{1}(x-y)+y\right\}^{h-1} \exp \left\{-\left(\frac{1}{\alpha_{1}}+\frac{1-\lambda_{1}}{\alpha_{1}}\right) y-\frac{\lambda_{1}}{\alpha_{1}} x\right\} & \text { if } x>y,\end{cases}
\end{aligned}
$$

for $x>0, y>0, h>0, l>0, \lambda_{1}>0, \lambda_{2}>0, \alpha_{1}>0$, and $\alpha_{2}>0$. For this distribution, the form of $R$ can be expressed as

$$
\begin{aligned}
R= & \frac{\lambda_{2}}{\Gamma(h) \Gamma(l) \alpha_{1}^{h} \alpha_{2}^{l}} \int_{0}^{\infty} x^{h-1} \exp \left\{-\left(\frac{1}{\alpha_{1}}+\frac{1-\lambda_{2}}{\alpha_{2}}\right) x\right\} \\
& \times \int_{x}^{\infty}\left\{\lambda_{2}(y-x)+x\right\}^{l-1} \exp \left\{-\frac{\lambda_{2}}{\alpha_{2}} y\right\} d y d x \\
= & \frac{1}{\Gamma(h) \Gamma(l) \alpha_{1}^{h} \alpha_{2}^{l}} \int_{0}^{\infty} x^{h-1} \exp \left(-\frac{x}{\alpha_{1}}\right) \int_{x}^{\infty} z^{l-1} \exp \left(-\frac{z}{\alpha_{2}}\right) d z d x \\
= & \frac{1}{\Gamma(h) \Gamma(l) \alpha_{1}^{h}} \int_{0}^{\infty} x^{h-1} \exp \left(-\frac{x}{\alpha_{1}}\right) \Gamma\left(l, \frac{x}{\alpha_{2}}\right) d x,
\end{aligned}
$$

where the transformation $z=\lambda_{2}(y-x)+x$ has been applied. By application of Lemma A.1 in the appendix, the integral in (6.2) can be calculated as

$$
\int_{0}^{\infty} x^{h-1} \exp \left(-\frac{x}{\alpha_{1}}\right) \Gamma\left(l, \frac{x}{\alpha_{2}}\right) d x=\frac{\alpha_{1}^{h+l} \alpha_{2}^{h}}{h\left(\alpha_{1}+\alpha_{2}\right)^{h+l}}{ }_{2} F_{1}\left(1, h+l ; h+1 ; \frac{\alpha_{2}}{\alpha_{1}+\alpha_{2}}\right) .
$$

Thus, the form of $R$ can be expressed in terms of the Gauss hypergeometric function as

$$
R=\frac{\alpha_{1}^{l} \alpha_{2}^{h}}{h B(h, l)\left(\alpha_{1}+\alpha_{2}\right)^{h+l}}{ }_{2} F_{1}\left(1, h+l ; h+1 ; \frac{\alpha_{2}}{\alpha_{1}+\alpha_{2}}\right) .
$$

If $l$ is an integer, then the hypergeometric term reduces to a finite sum, yielding

$$
R=\frac{\alpha_{2}^{h}}{h B(h, l)\left(\alpha_{1}+\alpha_{2}\right)^{h}} \sum_{k=0}^{l-1} \frac{(h)_{k}(1-l)_{k}}{(h+1)_{k} k !}\left(\frac{\alpha_{2}}{\alpha_{1}+\alpha_{2}}\right)^{k} .
$$




\section{Smith and Adelfang's bivariate gamma distribution}

Smith and Adelfang's [34] bivariate gamma distribution has the joint pdf specified by

$$
f(x, y)=K x^{\gamma_{1}-1} y^{\gamma_{2}-1} \exp \left(-\frac{x+y}{1-\eta}\right) \sum_{k=0}^{\infty} a_{k} I_{\gamma_{2}+k-1}\left(\frac{2 \sqrt{\eta x y}}{1-\eta}\right)
$$

for $x>0, y>0, \gamma_{1}>0, \gamma_{2}>0$, and $0<\eta<1$, where $K$ denotes the normalizing constant given by

$$
\frac{1}{K}=(1-\eta)^{\gamma_{1}} \Gamma\left(\gamma_{1}\right) \Gamma\left(\gamma_{2}-\gamma_{1}\right)
$$

Using the definition of $I_{m}(\cdot)$ given in Section 1, the corresponding form of $R$ can be expressed as

$$
\begin{aligned}
R=K \sum_{k=0}^{\infty} a_{k} \sum_{l=0}^{\infty} \frac{1}{l ! \Gamma\left(\gamma_{2}+l+k\right)}\left(\frac{\sqrt{\eta}}{1-\eta}\right)^{k+2 l+\gamma_{2}-1} \\
\quad \times \int_{0}^{\infty} x^{\gamma_{1}+l+\left(\gamma_{2}+k\right) / 2-1} \int_{x}^{\infty} y^{\gamma_{2}+l+\left(\gamma_{2}+k-1\right) / 2-1} \exp \left(-\frac{x+y}{1-\eta}\right) d y d x \\
=K \sum_{k=0}^{\infty} a_{k} \sum_{l=0}^{\infty} \frac{\eta^{\left(k+2 l+\gamma_{2}-1\right) / 2}(1-\eta)^{\left(\gamma_{2}-k-2 l+1\right) / 2}}{l ! \Gamma\left(\gamma_{2}+l+k\right)} J(k, l),
\end{aligned}
$$

where $J(k, l)$ denotes the integral

$$
J(k, l)=\int_{0}^{\infty} x^{\gamma_{1}+\left(k+2 l+\gamma_{2}-1\right) / 2-1} \exp \left(-\frac{x}{1-\eta}\right) \Gamma\left(\frac{k+2 l+3 \gamma_{2}-1}{2}, \frac{x}{1-\eta}\right) d x .
$$

Application of Lemma A.1 in the appendix shows that $J(k, l)$ can be expressed in terms of the Gauss hypergeometric function as

$$
\begin{aligned}
J(k, l)= & \frac{(1-\eta)^{\gamma_{1}+l+\left(\gamma_{2}+k-1\right) / 2} \Gamma\left(\gamma_{1}+2 \gamma_{2}+k+2 l-1\right)}{2^{\gamma_{1}+2 \gamma_{2}+k+2 l-2}\left(2 \gamma_{1}+\gamma_{2}+k+2 l-1\right)} \\
& \times{ }_{2} F_{1}\left(1, \gamma_{1}+2 \gamma_{2}+k+2 l-1 ; \gamma_{1}+l+\frac{\gamma_{2}+k+1}{2} ; \frac{1}{2}\right) .
\end{aligned}
$$

Substituting this into (7.3), one obtains the expression

$$
\begin{aligned}
& R= K(1-\eta)^{\gamma_{1}+\gamma_{2}} 2^{2-\gamma_{1}-2 \gamma_{2}} \\
& \times \sum_{k=0}^{\infty} a_{k} \sum_{l=0}^{\infty} \frac{\eta^{\left(k+2 l+\gamma_{2}-1\right) / 2} \Gamma\left(\gamma_{1}+2 \gamma_{2}+k+2 l-1\right)}{l ! 2^{k+2 l}\left(2 \gamma_{1}+\gamma_{2}+k+2 l-1\right) \Gamma\left(\gamma_{2}+l+k\right)} \\
& \quad \times{ }_{2} F_{1}\left(1, \gamma_{1}+2 \gamma_{2}+k+2 l-1 ; \gamma_{1}+l+\frac{\gamma_{2}+k+1}{2} ; \frac{1}{2}\right),
\end{aligned}
$$

a double infinite sum of terms involving the Gauss hypergeometric function. 


\section{Conclusions}

We have calculated the forms of $R=\operatorname{Pr}(X<Y)$ for six flexible families of bivariate gamma distributions. It would be of interest to emulate this work for other continuous bivariate distributions, including bivariate Laplace distributions, bivariate logistic distributions, and bivariate extreme value distributions. It would also be of interest to extend this work for continuous multivariate distributions. We hope to address some of these issues in a future paper.

\section{Appendix}

Some technical lemmas required for the calculations above are noted below.

Lemma A.1 (Gradshteyn and Ryzhik [7, equation (6.455.1)]). For $c>0, p>0$, and $\alpha+v>0$,

$$
\int_{0}^{\infty} x^{\alpha-1} \exp (-p x) \Gamma(\nu, c x) d x=-\frac{c^{\nu} \Gamma(\alpha+\nu)}{\nu p^{\alpha+\nu}}{ }_{2} F_{1}\left(\nu, \alpha+v ; \nu+1 ;-\frac{c}{p}\right)+\frac{\Gamma(\nu) \Gamma(\alpha)}{p^{\alpha}} .
$$

Lemma A.2 (Prudnikov et al. [31, Volume 2, equation (2.10.2.2)]). For $\alpha>0$ and $\beta>0$,

$$
\begin{aligned}
& \int_{0}^{a} x^{\alpha-1}(a-x)^{\beta-1} \Gamma(\nu, c x) d x \\
& \quad=a^{\alpha+\beta-1} \Gamma(\nu) B(\alpha, \beta)-v^{-1} a^{\alpha+\beta+\nu-1} c^{\nu} B(\beta, \alpha+\nu)_{2} F_{2}(\nu, \alpha+\nu ; \nu+1, \alpha+\beta+\nu ;-a c) .
\end{aligned}
$$

Lemma A.3 (Prudnikov et al. [32, Volume 3, equation (2.22.3.5)]). For $c>0$ and $\sigma>w$,

$$
\int_{0}^{\infty} x^{c-1} \exp (-\sigma x){ }_{2} F_{2}(a, b ; d, c ; w x) d x=\sigma^{-c} \Gamma(c){ }_{2} F_{1}\left(a, b ; d ; \frac{w}{\sigma}\right) .
$$

\section{Acknowledgments}

The author is grateful to Professor Samuel Kotz (George Washington University, USA) and to Professor Marianna Pensky (University of Central Florida, USA) for introducing him to this area of research. The author would also like to thank the referee and the editors for carefully reading the paper and for their great help in improving the paper.

\section{References}

[1] B. C. Arnold and D. Strauss, Bivariate distributions with exponential conditionals, J. Amer. Statist. Assoc. 83 (1988), no. 402, 522-527.

[2] A. M. Awad, M. A. Hamdan, and M. M. Azzam, Some inference results on $\operatorname{Pr}(X<Y)$ in the bivariate exponential model, Comm. Statist. Theory Methods 10 (1981), no. 24, 2515-2525.

[3] P. J. Becker and J. J. J. Roux, A bivariate extension of the gamma distribution, South African Statist. J. 15 (1981), no. 1, 1-12.

[4] K. C. Cherian, A bi-variate correlated gamma-type distribution function, J. Indian Math. Soc. (N.S.) 5 (1941), 133-144.

[5] E. Cramer and U. Kamps, The UMVUE of $P(X<Y)$ based on type-II censored samples from Weinman multivariate exponential distributions, Metrika 46 (1997), no. 2, 93-121. 
[6] A. Dussauchoy and R. Berland, Lois gamma à deux dimensions, C. R. Acad. Sci. Paris Sér. A-B 274 (1972), A1946-A1949 (French).

[7] I. S. Gradshteyn and I. M. Ryzhik, Table of Integrals, Series, and Products, Academic Press, California, 2000.

[8] R. C. Gupta and S. Subramanian, Estimation of reliability in a bivariate normal distribution with equal coefficients of variation, Comm. Statist. Simulation Comput. 27 (1998), no. 3, 675-698.

[9] R. D. Gupta and R. C. Gupta, Estimation of $\operatorname{Pr}\left(a^{\prime} x>b^{\prime} y\right)$ in the multivariate normal case, Statistics 21 (1990), no. 1, 91-97.

[10] D. D. Hanagal, Testing reliability in a bivariate exponential stress-strength model, J. Indian Statist. Assoc. 33 (1995), 41-45.

[11] - Note on estimation of reliability under bivariate Pareto stress-strength model, Statist. Papers 38 (1997), no. 4, 453-459.

[12] Estimation of reliability of a component subjected to bivariate exponential stress, Statist. Papers 40 (1999), no. 2, 211-220.

[13] P. K. Jana, Estimation for $P[Y<X]$ in the bivariate exponential case due to Marshall and Olkin, J. Indian Statist. Assoc. 32 (1994), no. 1, 35-37.

[14] Estimation of $P[Y<X]$ under a bivariate exponential stress-strength model, Frontiers in Probability and Statistics (Calcutta, 1994/1995) (S. P. Mukherjee, S. K. Basu, and Sinha B. K., eds.), Narosa, New Delhi, 1998, pp. 207-214.

[15] P. K. Jana and D. Roy, Estimation of reliability under stress-strength model in a bivariate exponential set-up, Calcutta Statist. Assoc. Bull. 44 (1994), no. 175-176, 175-181.

[16] E. S. Jeevanand, Bayes estimation of $P(X 2<X 1)$ for a bivariate Pareto distribution, The Statistician 46 (1997), no. 1, 93-99.

[17]__ Bayes estimation of reliability under stress-strength model for the Marshall-Olkin bivariate exponential distribution, IAPQR Trans. 23 (1998), no. 2, 133-136.

[18] J. P. Klein and A. P. Basu, Estimating reliability for bivariate exponential distributions, Sankhyā Ser. B 47 (1985), no. 3, 346-353.

[19] A. T. McKay, Sampling from batches, Journal of the Royal Statistical Society-Supplement 1 (1934), 207-216.

[20] S. P. Mukherjee and L. K. Saran, Estimation of failure probability from a bivariate normal stressstrength distribution, Microelectonics Reliability 25 (1985), 692-702.

[21] S. Nadarajah, Reliability for beta models, Serdica Math. J. 28 (2002), no. 3, 267-282.

[22] _ Reliability for extreme value distributions, Math. Comput. Modelling 37 (2003), no. 910, 915-922.

[23] _ Reliability for lifetime distributions, Math. Comput. Modelling 37 (2003), no. 7-8, 683688.

[24]_, Reliability for logistic distributions, Engineering Simulation 26 (2004), 81-98.

[25] _ Reliability for Laplace, Math. Probl. Eng. 2004 (2004), no. 2, 169-183.

[26] _ Reliability for some bivariate beta distributions, Math. Probl. Eng. 2005 (2005), no. 1, 101-111.

[27] S. Nadarajah and S. Kotz, Reliability for Pareto models, Metron 61 (2003), no. 2, 191-204.

[28] S. B. Nandi and A. B. Aich, A note on confidence bounds for $P(X>Y)$ in bivariate normal samples, Sankhyā Ser. B 56 (1994), no. 2, 129-136.

[29]_ A note on testing hypothesis regarding $P(X>Y)$ in bivariate normal samples, IAPQR Trans. 21 (1996), no. 2, 149-153.

[30] A. P. Prudnikov, Yu. A. Brychkov, and O. I. Marichev, Integrals and Series. Vol. 1, Gordon \& Breach Science Publishers, New York, 1986.

[31] , Integrals and Series. Vol. 2, Gordon \& Breach Science Publishers, New York, 1986.

[32] _ Integrals and Series. Vol. 3, Gordon \& Breach Science Publishers, New York, 1986. 
[33] N. Singh, MVUE of $\operatorname{Pr}(X<Y)$ for multivariate normal populations: an application to stressstrength models, IEEE Trans. Rel. 30 (1981), 192-193.

[34] O. E. Smith and S. I. Adelfang, Gust model based on the bivariate gamma probability distribution, Journal of Spacecraft and Rockets 18 (1981), 545-549.

Saralees Nadarajah: Department of Statistics, University of Nebraska, Lincoln, NE 68583, USA

E-mail address: snadaraj@unlserve.unl.edu 


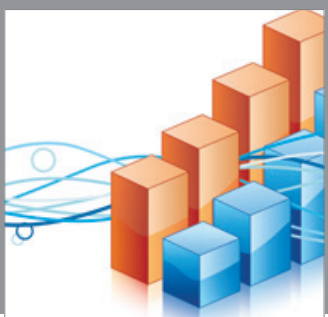

Advances in

Operations Research

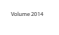

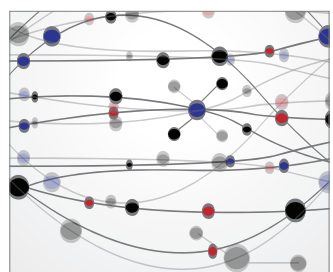

\section{The Scientific} World Journal
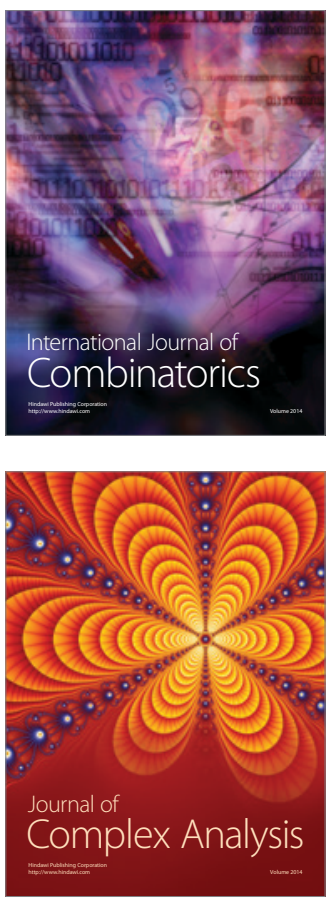

International Journal of

Mathematics and

Mathematical

Sciences
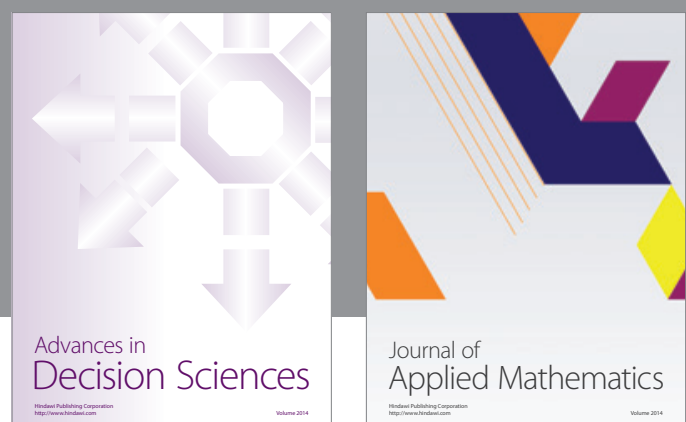

Journal of

Applied Mathematics
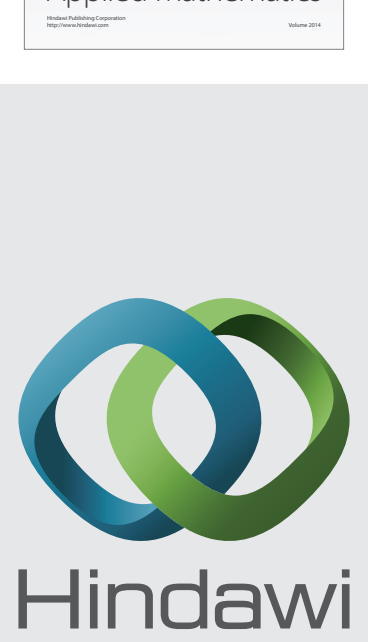

Submit your manuscripts at http://www.hindawi.com
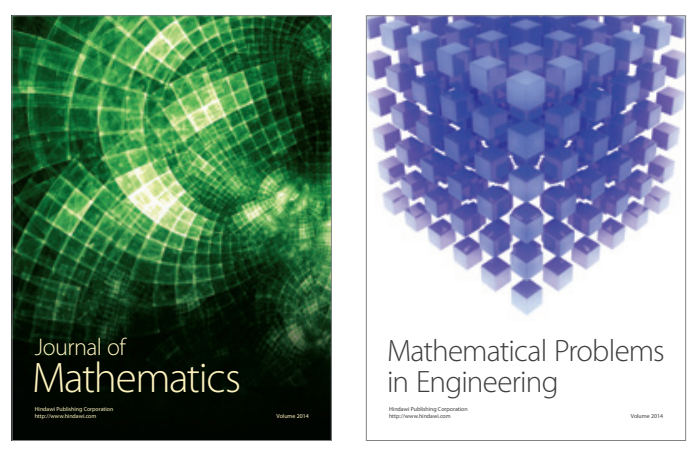

Mathematical Problems in Engineering
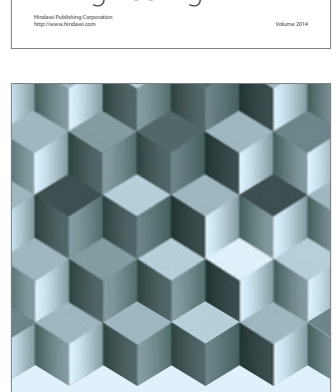

Journal of

Function Spaces
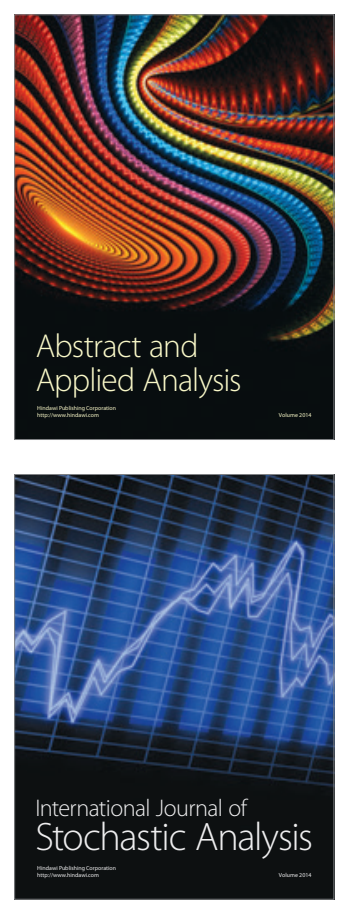

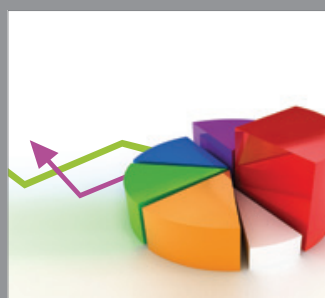

ournal of

Probability and Statistics

Promensencen
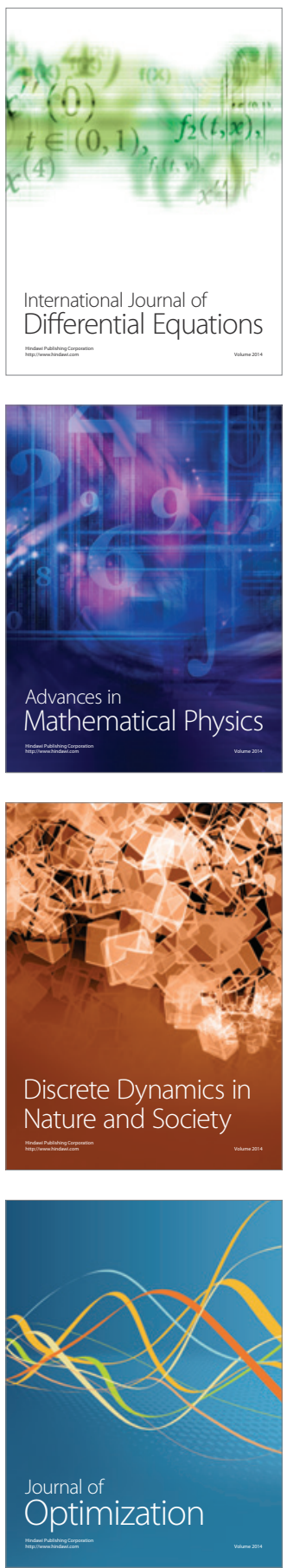